\title{
sciendo
}

\section{Coordination of Axial Trunk Rotations During Gait in Low Back Pain. A Narrative Review}

\author{
by \\ Jaap H. van Dieën ${ }^{1}$, Maarten R. Prins ${ }^{1,2,3}$, Sjoerd M. Bruijn ${ }^{1,4,5}$, Wen Hua Wu ${ }^{4,6}$, \\ Bowei Liang7, Claudine J.C. Lamoth ${ }^{8}$, Onno G. Meijer ${ }^{1,4}$
}

\begin{abstract}
Chronic low back pain patients have been observed to show a reduced shift of thorax-pelvis relative phase towards out-of-phase movement with increasing speed compared to healthy controls. Here, we review the literature on this phase shift in patients with low back pain and we analyze the results presented in literature in view of the theoretical motivations to assess this phenomenon. Initially, based on the dynamical systems approach to movement coordination, the shift in thorax-pelvis relative phase with speed was studied as a self-organizing transition. However, the phase shift is gradual, which does not match a self-organizing transition. Subsequent emphasis in the literature therefore shifted to a motivation based on biomechanics. The change in relative phase with low back pain was specifically linked to expected changes in trunk stiffness due to 'guarded behavior'. We found that thorax-pelvis relative phase is affected by several interacting factors, including active drive of thorax rotation through trunk muscle activity, stride frequency and the magnitude of pelvis rotations. Large pelvis rotations and high stride frequency observed in low back pain patients may contribute to the difference between patients and controls. This makes thorax-pelvis relative phase a poor proxy of trunk stiffness. In conclusion, thorax-pelvis relative phase cannot be considered as a collective variable reflecting the orderly behaviour of a complex underlying system, nor is it a marker of specific changes in trunk biomechanics. The fact that it is affected by multiple factors may explain the considerable between-subject variance of this measure in low back pain patients and healthy controls alike.
\end{abstract}

Key words: low back pain, coordination, gait, trunk, relative phase.

\section{Introduction}

During normal gait, the thorax and pelvis rotate more-or-less in the same direction around the vertical at low gait speed, and more-or-less in opposite directions at high speed, resulting in axial torsion of the lumbar spine (Crosbie et al., 1997; Wagenaar and Beek, 1992). In individuals with non-specific, chronic low back pain, the relative timing of these rotations appears to be different, with a reduced shift towards opposite rotations at higher speeds (Lamoth et al., 2002b; Selles et al., 2001). This phenomenon has received substantial attention in our own published work, and other published literature, but results have not been consistent and a thorough explanation

\footnotetext{
1 - Department of Human Movement Sciences, Faculty of Behavioural and Movement Sciences, Vrije Universiteit Amsterdam, and Amsterdam Movement Sciences, Amsterdam, The Netherlands.

2 - Research and Development, Military Rehabilitation Center 'Aardenburg', Doorn, The Netherlands.

3 - Institute for Human Movement Studies, HU University of Applied Sciences Utrecht, Utrecht, The Netherlands.

4 - Orthopaedic Biomechanics Laboratory, Fujian Medical University, Quanzhou, Fujian, PR China.

5 - Institute of Brain and Behaviour Amsterdam, Amsterdam, The Netherlands.

6 - Department of Orthopaedic Surgery, Second Affiliated Hospital of Fujian Medical University, Fujian, P.R. China.

7 - Department of Orthopaedics, Affiliated Dongnan Hospital of Xiamen University, Zhengzhou, Fujian, P.R. China.

8 - University of Groningen, University Medical Center Groningen, Center for Human Movement Sciences, Groningen The Netherlands.
} 
for the findings is still lacking. Here, we review the literature on relative timing between thorax and pelvis during gait in patients with low back pain and the theoretical motivations to assess this relative timing. We analyze the results presented in literature in view of these motivations.

The initial impetus for studying relative timing of thorax and pelvis rotations came from the idea that the shift in relative timing is a selforganizing transition (Wagenaar and Beek, 1992), as understood in the dynamical systems approach to movement coordination (Haken et al., 1985). We briefly summarize this approach and show that the empirical observations reported in literature do not support the interpretation of this phenomenon as a self-organizing transition. Subsequent emphasis in the literature implicitly shifted from interpretation based on the dynamical systems theory to one based on biomechanics, where the change in relative timing with LBP was assumed to emerge from the mechanical effects of changes in trunk muscle activity, specifically an increased trunk stiffness. We therefore review the relation between trunk mechanical properties and relative phase. We show that also this motivation turns out flawed, since relative timing is affected by several interacting factors, making thorax-pelvis timing a poor proxy of the trunk's mechanical properties. But, to set the stage, we will first briefly define measures of relative timing of segment rotations.

\section{Relative timing and relative phase}

Thorax and pelvis rotations during gait are more-or-less periodic signals, completing one period each gait cycle (Figure 1, upper panel). Terms like 'in the same direction' or 'en bloc' (Hulbert et al., 2015) versus 'in opposite directions' or 'counterrotation' (Selles et al., 2001) describe the timing differences between the cycles of thorax and pelvis, but not in a very precise manner. A more exact description can be obtained by cross-correlating these signals, to obtain the time lag at which maximal correlation occurs (Wagenaar and Beek, 1992). However, describing the difference in timing in absolute time does not sufficiently capture the intersegmental coordination, because the stride cycle time varies between and within subjects. For example, a shift in time of 0.3 seconds describes a different intersegmental coordination pattern if the stride time is 1.3 seconds (i.e., more towards 'en bloc') than if the stride time is 0.7 seconds (i.e., more towards counterrotation). Relative phase measures, which have been used in studying selforganizing transitions (Haken et al., 1985), account for the effect of cycle duration and may quantify the shift in timing of pelvis and thorax rotations with increasing gait speed (Huang et al., 2011; Huang et al., 2010; Lamoth et al., 2006a; Lamoth et al., 2002b; Prins et al., 2016, 2019a; Prins et al., 2019b; Selles et al., 2001; Wagenaar and Beek, 1992).

The continuous relative phase expresses the timing difference between two periodic signals as a phase angle [16]. If the orientation of a segment follows a periodic pattern, its state at any point in time is fully specified by its orientation and is angular velocity. Consider for example the zero crossings of a periodic signal. These signify an orientation angle of zero, but in the same periodic signal, the angular velocity at such a point can be positive, when the signal moves from a negative to a positive orientation, or negative when it moves from a positive to a negative orientation. Hence both variables are needed to fully describe the kinematics and more variables (higher order derivatives e.g. acceleration, jerk) are only needed for non-periodic signals. Therefore, to represent the kinematic state, the time series of the orientation angle of a body segment during a cyclic movement like walking can be plotted against its angular velocity. This yields a more or less round shape (if the amplitudes of the signals are normalized (Lamb and Stöckl, 2014)), with a complete orbit during each stride cycle. The angle relative to the coordinate system of this phase plot expresses the phase in the cycle at each point in time (Figure 1, middle panel). The difference between the phase of thorax rotations and pelvis rotations at any given point in time is defined as the thorax-pelvis relative phase (Figure 1, lower panel).

Since segmental movements during gait do not follow a perfect sinusoidal pattern, a continuous relative Fourier phase method was introduced to calculate the relative phase between thorax and pelvis rotations (Lamoth et al., 2002a). With this method, the time signal is decomposed into its constituent frequencies with corresponding phase and amplitude, and relative phase is calculated between segments at the stride frequency. This filters out possible artefacts in 
relative phase due to higher dominant frequencies (Lamoth et al., 2002a), which are present in segment rotations during gait (Lamoth et al., 2002a; van den Hoorn et al., 2012). A difference in phase or relative phase of zero degrees indicates that the two segments move perfectly in-phase. A relative phase of $+/-180$ degrees means that the two segments are perfectly out-of-phase, moving in opposite directions.

\section{Thorax-pelvis relative phase in gait}

As described above, in healthy gait, the relative phase between thorax and pelvis rotations in the transverse plane shifts from close to inphase at low gait speeds to more out-of-phase at high speeds. Typical examples of this pattern are shown in Figure 2. At low speeds the thorax closely follows the pelvis, at a relative phase of approximately -20 degrees. With increasing speed the phase lag increases, resulting in a more out-ofphase coordination pattern, with thorax-pelvis relative phase typically reaching values around 140 degrees at $1.5 \mathrm{~m} / \mathrm{s}$ (Lamoth et al., 2002a).

The first studies on thorax-pelvis relative phase during gait did not relate the timing of these segments to the pendular movements of the arms and legs. Hence, it was not known how changes in thorax-pelvis relative phase were related to the gait cycle. Obviously, the legs tend to move out-of-phase with respect to each other at all gait speeds. At (very) low gait speed, generally below $0.5 \mathrm{~m} / \mathrm{s}$, the left and right arm tend to move in-phase, making two complete cycles each stride cycle (2:1 ratio with the legs) (Donker et al., 2001; Wagenaar and van Emmerik, 2000). At higher speeds, the arms tend to move almost out-ofphase relative to each other and to the ipsilateral leg (e.g., while the left arm is swinging forward, the left leg and right arm swing backward) (Huang et al., 2010).

In healthy subjects, the timing of the thorax relative to the legs is relatively constant over walking speeds, with the thorax rotating almost perfectly out-of-phase with the pendular movements of the legs (i.e., the right side of the thorax moves forward when the right leg moves backward). At low gait speed, the pelvis rotates more-or-less out-of-phase with the pendular movements of the legs (approximately -110 degrees at $0.56 \mathrm{~m} / \mathrm{s}$ ), with the pelvis lagging the legs. When speeding up, pelvis and leg start to move more in-phase to approximately -50 degrees at $1.44 \mathrm{~m} / \mathrm{s}$ (Bruijn et al., 2008; Huang et al., 2010). Hence, the change in thorax-pelvis relative phase as a function of gait speed is associated with a change in pelvis-leg timing rather than a change in thorax-leg timing.

\section{Thorax-pelvis relative phase and low back pain}

The first studies that reported altered thorax-pelvis relative phase during gait in chronic low back pain measured patients and healthy controls over a range of walking speeds (Lamoth et al., 2002b; Selles et al., 2001). Only at relatively high walking speeds, from $1.06 \mathrm{~m} / \mathrm{s}$ upward, a difference was observed in thorax-pelvis relative phase between groups. At low gait speeds, thorax and pelvis rotated more-or-less in-phase in both groups. At higher speeds, the lag in thorax rotations, resulting in a more out-of-phase coordination pattern, was larger in healthy controls than in patients resulting in significantly more in-phase (or less out-of-phase) thorax-pelvis relative phase in low back pain patients than controls (Figure 3). Lamoth et al. (2002b). reported that at $1.5 \mathrm{~m} / \mathrm{s}$, a relatively high walking speed, the thorax-pelvis relative phase of healthy subjects was approximately -140 degrees and in chronic low back pain patients it was approximately -110 degrees (Figure 3 ). In most previous studies on relative timing of thorax and pelvis rotations pelvis-thorax relative phase (pelvis timing relative to the thorax) was reported. Because thorax rotations lag pelvis rotations, the pelvis appears to be an important driver of thorax rotations (and not the other way around). Hence, in this manuscript we choose to report thorax timing relative to the pelvis by converting all these values to thorax-pelvis relative phase (thorax timing relative to the pelvis) by multiplying these values by -1 . In words, at high walking speeds the axial thorax rotations were lagging pelvis rotations in both groups, and this delay was smaller in low back pain patients: a more in-phase coordination (Selles et al., 2001)..

Although thorax-pelvis relative phase during gait has been found to be significantly affected by low back pain in some studies or in some conditions within studies (Huang et al., 2011; Lamoth et al., 2006a; Lamoth et al., 2006b; Lamoth et al., 2002b; Selles et al., 2001), a considerable variance between subjects (see also Figure 2), and overlap between low back pain 
patients and controls was present. Moreover, some studies did not report significant betweengroup differences (Prins et al., 2016; Seay et al., 2011a; Seay et al., 2011b). In addition, we know from own experience that negative publication bias has led to not reporting null findings. Nevertheless, a recent review concluded that the finding of in-phase coordination is relatively robust when compared to other aspects of trunk kinematics in gait that are mostly not different between patients and controls (Koch and Hansel, 2018). In addition, it is worth noting that more inphase thorax-pelvis coordination has been observed in other pathologies as well, including Parkinson's disease (Van Emmerik et al., 1999), stroke (Van Criekinge et al., 2017), cerebral palsy (Tavernese et al., 2016), pregnancy-related pelvic girdle pain (Wu et al., 2008a), ankylosing spondylitis (Mangone et al., 2011), and transfemoral amputation (Russell Esposito and Wilken, 2014).

In low back pain patients, the timing of axial pelvis rotations relative to the pendular movements of arms and legs is similar to that in healthy controls (Huang et al., 2011; Prins et al., 2016). However, unlike in healthy controls, the timing of thorax rotations relative to the legs changes with gait speed, towards less out-ofphase thorax-leg coordination at higher speeds (Figure 3) (Huang et al., 2011; Prins et al., 2016). In other words, more in-phase thorax-pelvis relative phase in low back pain is associated with altered timing of thorax rotations relative to the gait cycle as defined by leg movement. Figure 3 depicts this effect of speed on timing of pelvis rotations relative to the gait cycle and the effect of pathology on timing of thorax rotations relative to the gait cycle.

The relative inconsistency of the literature regarding differences in thorax-pelvis relative phase between patients and controls may be due to the large between-subject variance in this parameter. Substantial variance between subjects was already pointed out by Lamoth et al. (2002b), who reported that about one third of the patients walked in-phase at all speeds tested $(0.33-1.50$ $\mathrm{m} / \mathrm{s}$ ), about one third showed an effect of speed on relative phase that was clearly smaller than in healthy controls and about one third showed a normal shift towards out-of-phase rotations at higher speeds. Prins et al (2016) found no significant difference in relative phase between patients and controls walking $1.08 \mathrm{~ms} / \mathrm{s}$. In their controls, thorax-pelvis relative phase was -101 (SD 39) degrees; in their patients, it was -83 (SD 26) degrees, suggesting similar coefficients of variation in both groups of about $30-40 \%$. These findings indicate that less out-of-phase phase thorax-pelvis coordination at high gait speeds, is present in some but not all patients, which may be related to the heterogeneity of motor control changes in low back pain patients (van Dieën et al., 2019b). As such, these changes could still be informative when aiming to identify patients with (specific) motor control issues to provide more targeted treatment (van Dieën et al., 2019a).

\section{Dynamical systems approach to movement coordination}

The shift in relative timing of pelvis and thorax rotations with increasing gait speed first caught the interest of researchers inspired by Haken's (1985) dynamical systems approach (e.g.Lamoth et al., 2002a; Selles et al., 2001; Van Emmerik et al., 1999; Wagenaar and Beek, 1992). This approach aims to phenomenologically model the behaviour of movement systems, composed of a large number of interacting neural, vascular, muscular, and skeletal elements (Beek et al., 1995). The orderly behaviour of the complex dynamical system is reflected in its collective variables. Behaviour of the collective variables emerges given the values of one or more 'control parameters'. These affect the number and/or kind of coordination modes reflected by the collective variable(s). The dynamical systems approach attempts to model the system's dynamics, without explicit representation of the components of the system and of the interactions between these components. Abstract models of the dynamics of the collective variables allow prediction of coordinated movement behaviour under varying conditions. A major impetus to apply the dynamical systems theory in movement science was formed by experimental and modelling work on the coordination of rhythmic finger movements (Haken et al., 1985; Kelso, 1984; Kelso, 1981). The focus of this work was the transition in relative timing between two periodically moving index fingers, induced by increasing movement frequency. When moving the fingers out-of-phase, increasing the movement frequency causes a transition to in-phase movement. A crucial step in 
modelling is to identify the collective variable(s) and the control parameter(s) that capture the behaviour of the system. The hallmarks of a collective variable are that it shows abrupt transitions between stable modes of coordination with a change in what is called an order parameter and that it shows increased variability around these transitions. The relative phase of the finger movements fulfilled these criteria with movement frequency as the control parameter. Subsequent modelling work based on these findings was successful in describing many features of coordination between fingers, but also between for example an arm and a leg (Kelso and Jeka, 1992), and an arm and a visual stimulus (Byblow et al., 1995), and between leg movements of two individuals (Schmidt et al., 1990). At first sight, the shift in relative timing between thorax and pelvis with increasing gait speed could be seen as another example of such a phase transition. If correct, that could provide critical information on the coordination of gait. But, can this shift in timing indeed be considered a phase transition and can the relative phase and gait speed be considered respectively the collective variable and control parameter of gait coordination?

Thorax-pelvis relative phase during gait has consistently been reported as a function of gait speed (Huang et al., 2011; Huang et al., 2010; Lamoth et al., 2002a; Lamoth et al., 2006a; Lamoth et al., 2002b; Selles et al., 2001; Van Emmerik et al., 1999; Wagenaar and Beek, 1992). The example of finger movements described above might, however, suggest that the related variable stride frequency is a more likely candidate control parameter. Since stride frequency generally increases with speed, the relation between speed and relative phase could be spurious. Huang et al. (2010; 2011) manipulated both stride frequency and gait speed by imposing small, normal and large steps at three fixed speeds imposed by a treadmill. In these studies, the expected change in relative phase with gait speed was confirmed, but the relationship between stride frequency and thorax-pelvis coordination was inconsistent. At each speed, higher stride frequencies (smaller steps) resulted in more in-phase thorax-pelvis coordination. At each step length, higher frequencies (higher speed) resulted in more outof-phase thorax pelvis coordination
(Huang et al., 2011; Huang et al., 2010). These results indicate that thorax-pelvis relative phase is more affected by gait speed than stride frequency (see Figure 2) and hence it makes sense to consider gait speed as a candidate control parameter. However, when doing so, the shift in relative phase between pelvis and thorax rotations does not fulfil the criteria for a phase transition. First as was observed already early on (Lamoth et al., 2002b), the transition in timing occurs gradually and not abruptly (see Figure 2), which accidently also applies when analysing relative phase as a function of stride frequency. This is in stark contrast with, for example, the changes in coordination at the transition from walking to running (Lamoth et al., 2009). In addition, Lamoth et al. (2002a) showed that no systematic increase in the variability of the relative phase occurred around that previous findings reporting such an increase were likely confounded by not using the Fourier phase. Other criteria for self-organizing transitions, the presence of critical slowing down and hysteresis have to our knowledge also not been confirmed for the shift in thorax-pelvis relative phase during gait. These findings indicate that the relative phase between pelvis and thorax rotations cannot be considered a collective variable as defined in Haken's understanding of self-organizing transitions. This may explain why no formal modelling of thorax-pelvis relative phase within the dynamical systems framework appears to have been attempted and why in publications explanations of the empirical findings were instead given in terms of the effects of trunk muscle activity on trunk mechanics.

\section{Biomechanics}

The first studies reporting that chronic low back pain patients demonstrate less out-ofphase thorax-pelvis coordination at higher walking speeds (Lamoth et al., 2002b; Selles et al., 2001) were inspired by findings of the same phenomenon in some patients with stroke (Wagenaar and Beek, 1992; Wagenaar and van Emmerik, 1994) and, more consistently, in patients with Parkinson's disease (Wagenaar and van Emmerik, 1994). Patients with Parkinson's disease have difficulty with many functional tasks involving axial rotation of the trunk (Lakke, 1985). 

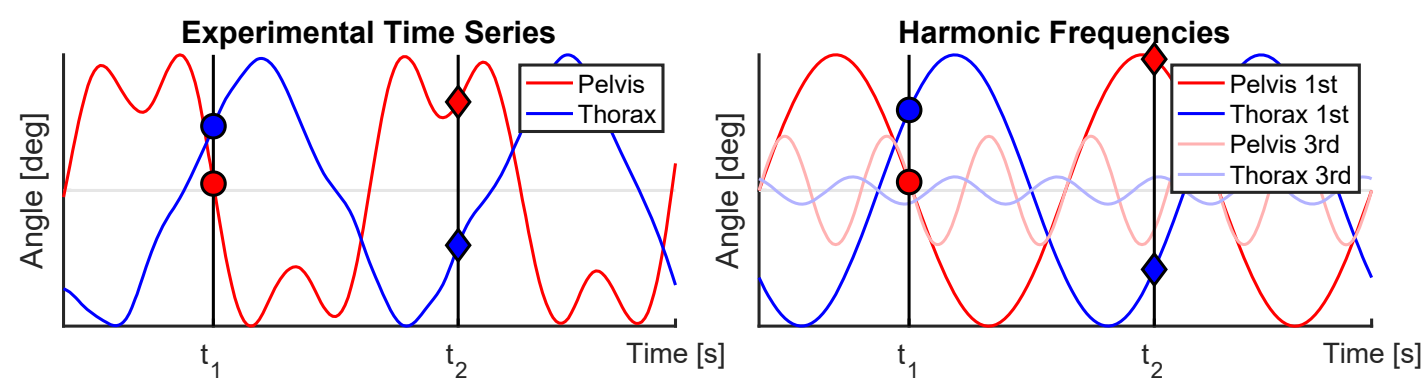

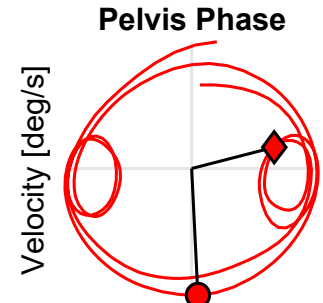

Angle [deg]

$t_{1}$ Relative Phase

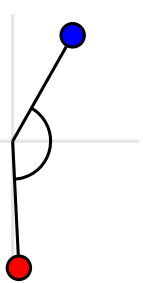

Figure 1

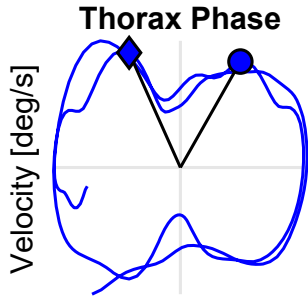

Angle [deg]
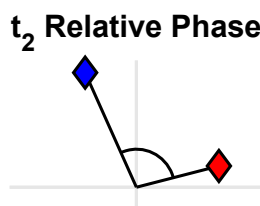

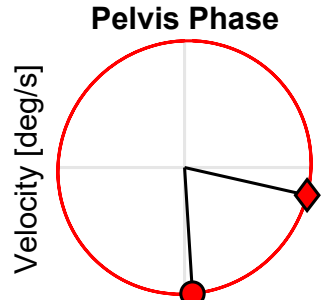

Angle [deg]

$t_{1}$ Relative Phase

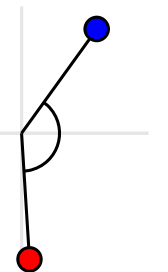

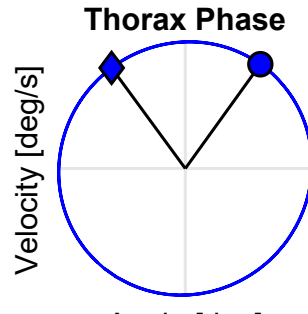

Angle [deg]
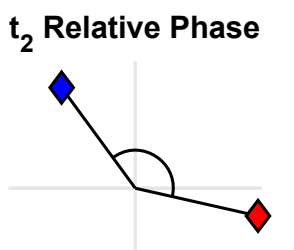

Thorax-pelvis relative phase at relatively high walking speed

The figures left from the midline represent realistic thorax-pelvis rotations at a walking speed of $\pm 1.5 \mathrm{~m} / \mathrm{s}$. The figures on the right display the harmonic frequencies of the time-series separated using a Fourier transform (time domain to frequency domain) and inverse Fourier Transform (harmonic frequency content to time domain).

Upper panel: Time-series of thorax-and pelvis rotations of two complete periods for each signal. The black line with two circles represents an arbitrary point in time ' $t 1^{\prime}$, the black line with two diamonds ' $2^{2}$ '.

Middle panel: Phase plots of the pelvis (blue) and thorax (red). The angles displayed in the upper panel are now plotted against the angular velocities of the same signals. The presented phase plots complete two orbits, one per stride cycle. $t_{1}$ and $t_{2}$ from the upper panel are displayed in this panel as well, representing the same points in time. Note that the left two figures are not perfectly round, which is mainly caused by the higher harmonic frequency content in the time series (causing a loop in the left and right side of the pelvis phase plot and a dent on the upper and lower side of the thorax phase plot).

Lower panel: The angle between the phase of the thorax and pelvis at $t_{1}$ and $t_{2}$ : thorax-pelvis relative phase. Note that thorax-pelvis relative phase is different between $t 1$ and $t 2$ in the left two figures, which is mainly the result of higher harmonics, and identical in the right two figures. 


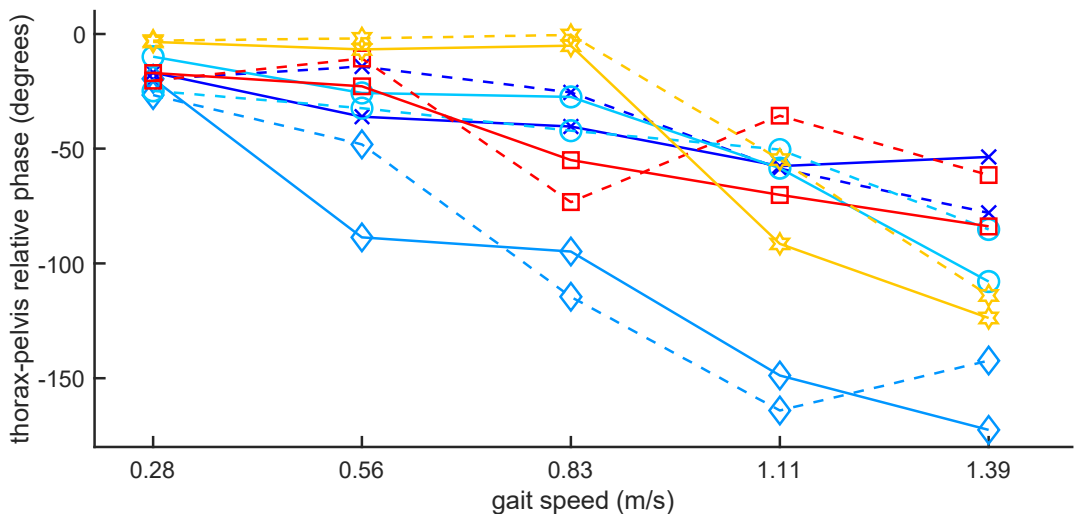

Figure 2

Thorax-pelvis relative phase as a function of gait speed in 5 healthy subjects.

Solid lines represent normal walking, dashed lines represent walking with large steps, i.e. at a lower than normal stride frequency. Corresponding colors and symbols indicate the same subject. The data illustrate the between-subject variance in the speed-relative phase relationship and the gradual decrease in relative phase with speed. The fact that relative phase decreases with speed in spite of the concomitant increase in stride frequency and the consistency of the relationships between normal and large steps in the same subjects indicate that gait speed affects the relative-phase more than stride frequency. Data from (Liang et al., 2014).

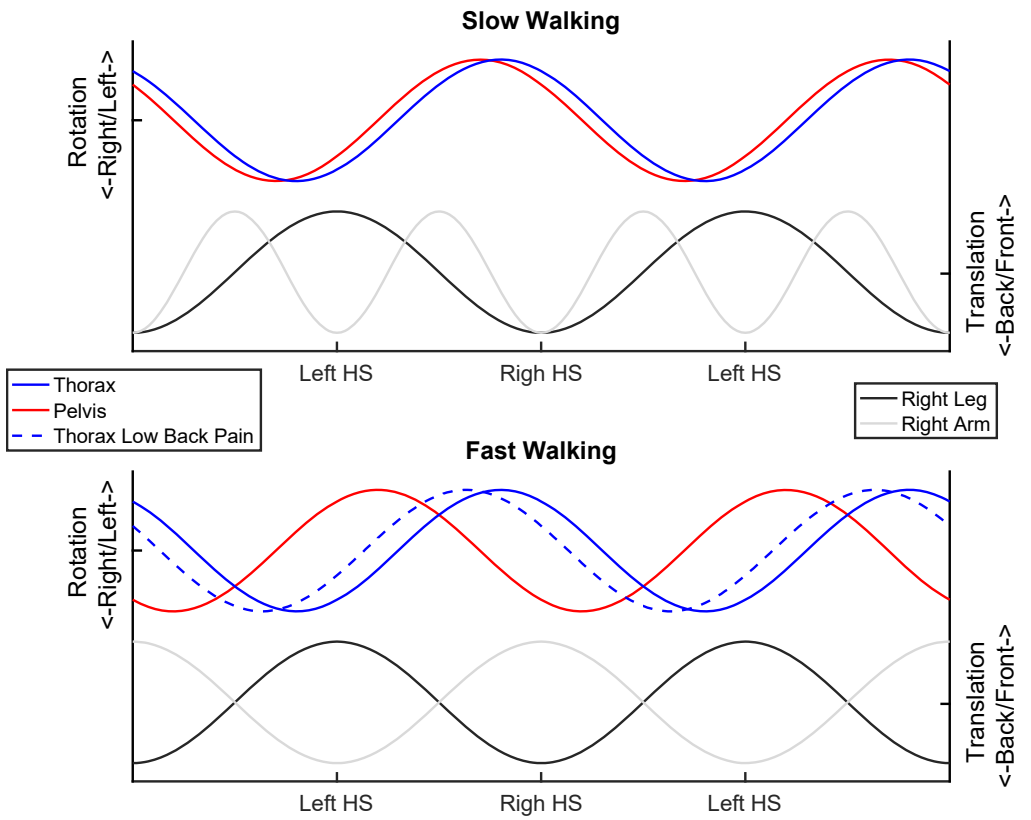

Figure 3

Relative timing of thorax and pelvis rotations and arm and leg swing during slow and fast walking in low back pain patients and healthy controls.

The first harmonic frequency content of each time series is displayed. The timing of most displayed signals is similar between low back pain patients and healthy controls. Hence, the pelvis, arms and legs are represented by single lines in both plots, as is the thorax in the upper plot. In the lower plot, the thorax of low back pain patients is represented by a dotted line. At low gait speed, thorax and pelvis rotate more-or-less out-of-phase with the pendular movements of the legs. At high gait speed the timing of the pelvis becomes more in-phase with the legs. In healthy controls, the timing of thorax rotations relative to the legs is relatively constant. In low back pain patients, the timing of thorax rotations shifts towards more in-phase with the legs with increasing gait speed, resulting in less out-of-phase thoraxpelvis coordination compared to healthy controls.

HS = Heel Strike 
Model Inputs
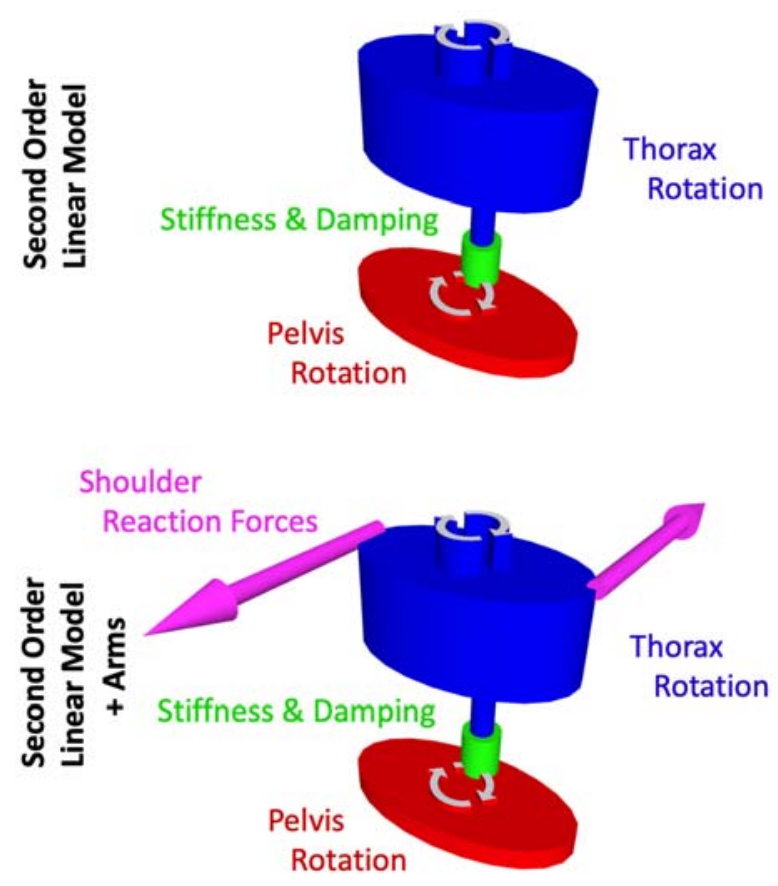

Frequency Response
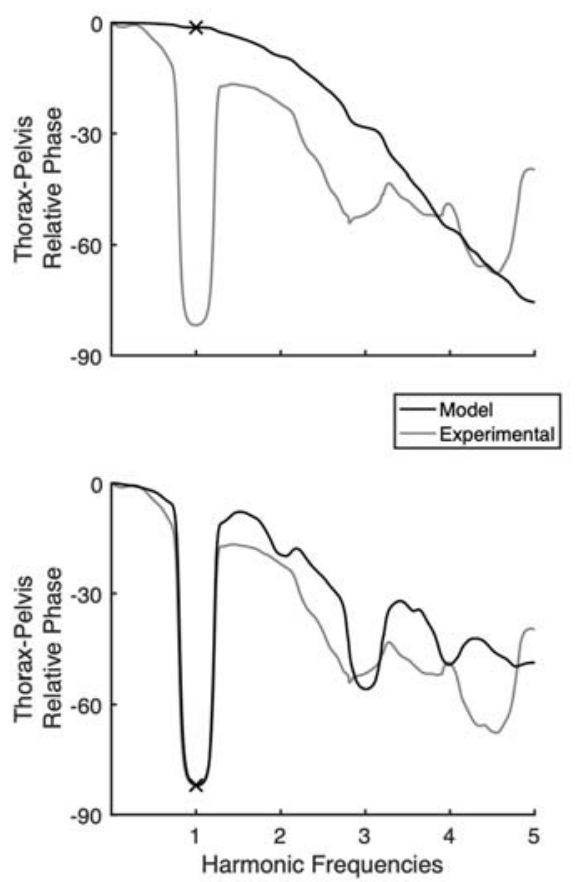

Figure 4

Forward dynamic models of the trunk that have been used to gain insight in mechanisms that affect thorax-pelvis coordination during gait.

Left: Two forward dynamic second order linear models that predict time series of thorax rotations from pelvis rotations, trunk stiffness and damping and shoulder reaction forces. Right: The frequency response function of each model when provided with actual gait data (black) compared to the actual frequency response function of the provided data (gray). Thorax-pelvis relative phase as defined in this review relates to the phase of the frequency response function at the first harmonic frequency (i.e., the stride frequency), indicated with an ' $x$ ' in each figure. The arms have a significant effect on thorax-pelvis relative phase, particularly at the first harmonic frequency. Hence, a second order linear model without the driving force of the arms does not appear a valid model of thorax-pelvis coordination during gait. 


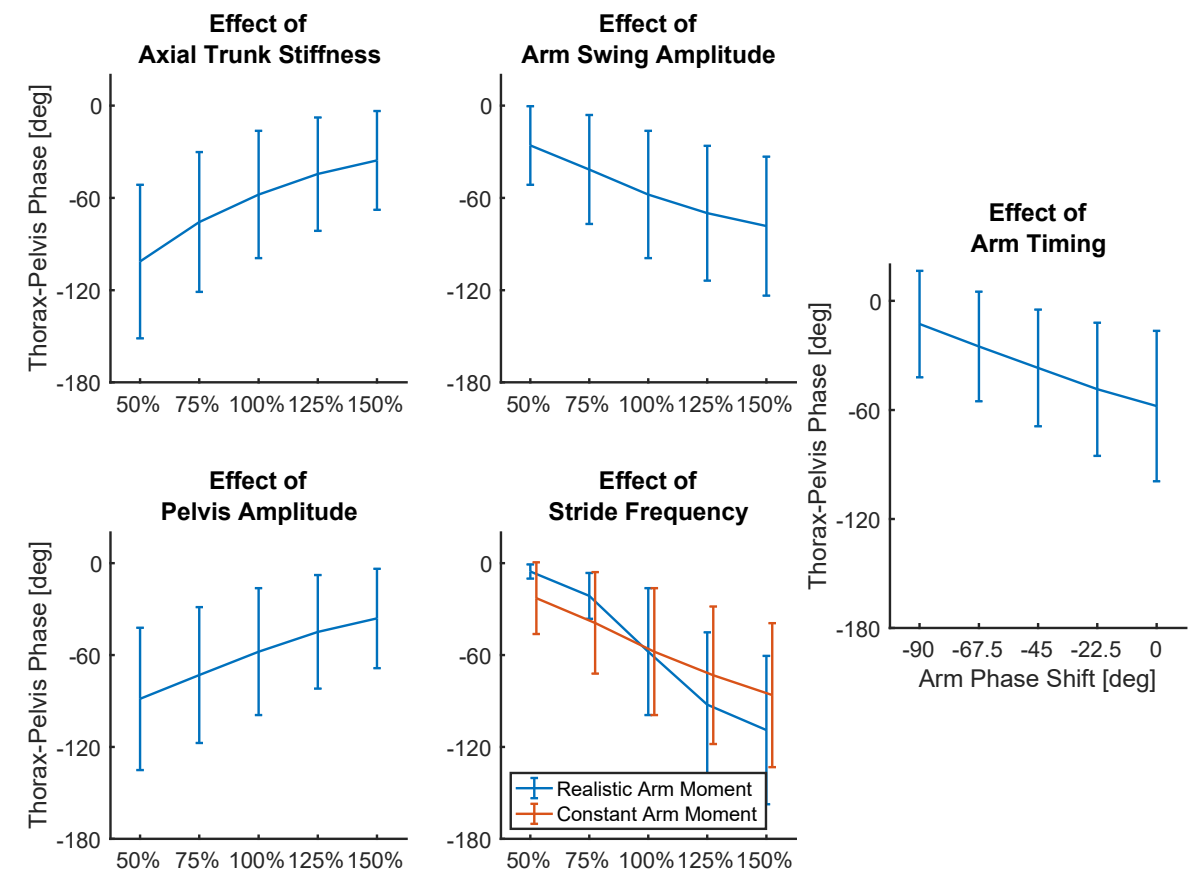

Figure 5

Effects of five mechanisms affecting thorax-pelvis relative phase during gait, predicted by a forward dynamic model.

Each figure displays the mean thorax-pelvis relative phase (vertical y-axis) of 30 subjects for different values of each of the five control parameters. Error bars indicate standard deviations. In the left four figures, the values at $100 \%$ on the $x$-axis correspond to actual experimental values of that parameter. Increasing stride frequency with a constant amplitude of arm swing, would increase shoulder reaction forces, which increases the effect on thorax-pelvis timing ('Realistic Arm Moment') compared to a simulation where this effect is neglected ('Constant Arm Moment'). In the right figure, the lag of 0 degrees indicates the average experimental timing of arm swing relative to the pelvis, negative phase shifts indicate a shift of arm swing timing relative to the experimental pelvis rotations, resulting in more in-phase movement relative to the pelvis. Details about the methods resulting in this figure are described in the Appendix.

During these tasks, they rotate the thorax and pelvis simultaneously, i.e., more 'en bloc' (Hulbert et al., 2015), which could be related to increased axial trunk stiffness (Wagenaar and van Emmerik, 1994). Also, in the studies on chronic low back pain, increased axial trunk stiffness was suggested as a possible cause for altered thoraxpelvis timing. However, rather than a symptom, this was suggested to be a local adaptation described as protective guarding or splinting (Lamoth et al., 2002b; Selles et al., 2001). While not in contradiction with the dynamical systems theory, these explanations attempt to attribute changes in coordination to specific components of the underlying system and do so in explicit mechanical terminology. In the next section, we 
will review mechanisms that may cause changes in thorax-pelvis relative phase.

Mechanisms that may underlie the more in-phase thorax-pelvis coordination in patients with low back pain as compared to healthy controls have been addressed in observational studies, by experimental manipulations of potential mechanisms, and finally in modelling studies. These studies will be reviewed in the subsequent subsections.

\section{Observational studies}

Observational studies have helped to identify possible mechanisms for more in-phase thoraxpelvis relative phase during gait. In a systematic review describing six case-control studies that measured electromyography of trunk muscles during speed-controlled gait, a higher erector spinae activity in low back pain patients was reported (Ghamkhar and Kahlaee, 2015). These conclusions should be interpreted with caution because electromyographical signals are difficult to relate to actual muscle activity and/or generated force (Staudenmann et al., 2010) especially if contractions are painful (GravenNielsen et al., 2002), although the effect of pain on trunk muscle activation appears to be limited in low back pain (Ng et al., 2002a; $\mathrm{Ng}$ et al., 2002b). Among the studies reviewed (Ghamkhar and Kahlaee, 2015), only one reported thorax-pelvis relative phase and found more in-phase coordination at higher gait speeds in low back pain (Lamoth et al., 2006b). However, in this study, the shapes of the EMG patterns and not their amplitudes were assessed. Overall, the review results (Ghamkhar and Kahlaee, 2015) indicate that increased trunk muscle activity may be present in patients with low back pain. This would likely result in increased trunk stiffness in these patients (Cholewicki et al., 1999; GardnerMorse and Stokes, 1998; Gardner-Morse and Stokes, 2001), which could be a cause of the more in-phase coordination.

Wu et al. (Wu et al., 2008b) observed that patients with pelvic girdle pain walked with larger axial pelvis rotations than healthy controls and Huang et al. (2011) found similar results in patients with lumbar disc herniation. Like chronic low back pain patients, both these patient groups walked less out-of-phase at higher speed compared to healthy controls (Wu et al., 2008a), although in patients with lumbar disc herniation this was significant only when walking with large steps (Huang et al., 2011). Also, Prins et al. (2016) reported an association between less out-of-phase thorax-pelvis coordination in chronic low back pain patients and larger pelvis amplitudes. Although the pathologies are different, these findings might point at a common mechanism.

Finally, low back pain patients have been reported to walk with a higher stride frequency at a given speed in multiple studies (Lamoth et al., 2006a; Vogt et al., 2001). Only one of these studies reported thorax-pelvis relative phase (Lamoth et al., 2006a), which was less out-of-phase in patients than in controls, possibly suggesting a causal relation of stride frequency with thorax-pelvis relative phase.

\section{Experimental studies}

Although observations can help to identify possible mechanisms that result in more in-phase thorax-pelvis coordination, experimental manipulation can provide more insight into the causality of the relationship between these outcomes. To evaluate if increased axial trunk stiffness would result in more in-phase thoraxpelvis coordination, Wu et al. (2014) studied the effect of voluntary abdominal co-contraction and of an orthopaedic abdominal brace during gait in healthy volunteers. The abdominal brace did result in more in-phase thorax-pelvis coordination, as observed in low back pain patients. However the timing of pelvis rotations relative to the legs changed, and the rotational amplitude of the trunk decreased, which both are in contrast with observations in patients (Huang et al., 2011; Lamoth et al., 2002b; Prins et al., 2016). These results would suggest that although increased trunk stiffness does affect thorax-pelvis relative phase, this might not be the mechanism causing more in-phase rotations in low back pain patients. Moreover, in a study in which axial perturbations of the spine were imposed during gait, no significant difference in the effect of these perturbations was observed between low back pain patients and controls (Prins et al., 2016). This also held for those patients who showed more inphase thorax-pelvis coordination, which suggests that trunk stiffness did not cause the change in coordination.

Prins et al. (2019b) manipulated the amplitude of pelvis rotations during gait of healthy subjects by asking them to walk with 
small, normal and large pelvis rotations at fixed speed and a metronome-imposed stride frequency. Walking with large pelvis rotations resulted in more in-phase thorax-pelvis coordination, hence, the relation between large pelvis rotations and in-phase pelvis thorax coordination in patients with chronic low back pain could be causal.

As discussed in section 5, stride frequency was manipulated in low back pain patients and healthy controls by Huang et al. $(2011 ; 2010)$ by imposing small, normal and large steps at fixed gait speeds. In these studies, a higher stride frequency (small steps) resulted in more in-phase thorax-pelvis coordination (Huang et al., 2011; Huang et al., 2010), with a larger effect in low back pain patients. This could indicate that a higher stride frequency contributes to the inphase thorax-pelvis coordination in patients with chronic low back pain (Huang et al., 2011).

\section{Modelling studies}

The relation between thorax-pelvis relative phase and stride frequency (i.e., more in-phase with higher frequency) observed in the studies of Huang et al. $(2011 ; 2010)$ was opposite to what was to be expected from second order linear dynamics (i.e. more out-of-phase at higher frequencies) [36]. Huang et al. (2010), therefore, stated that the rotations of the thorax relative to the pelvis are actively driven, and not determined by the passive dynamics of the system. A passive second order model of the trunk would consist of two rigid segments (thorax and pelvis), connected by a torsion spring and a damper (Figure 4). If the rotation of one segment is imposed in such a system, the phase of the other segment will lag the driving segment. Because thorax rotations lag pelvis rotations, the pelvis would appear to drive thorax rotations during gait. The thorax-pelvis relative phase would then depend on (1) trunk stiffness, becoming more in-phase with higher stiffness, and (2) the frequency of the pelvis rotation (i.e., the stride frequency), becoming more out-of-phase with increasing frequency, in contrast to what Huang et al. (2011; 2010) observed experimentally. Although second order linear models have been used for the mechanical behaviour of the spine in gait studies (Kubo et al., 2006; Prins et al., 2019a), there are two issues that, combined, complicate the interpretations. First, the arms exert a considerable pull on the thorax via the reaction forces in the shoulders (Bruijn et al., 2008; Ford et al., 2007). If arm swing is added to the model, this would affect its behaviour. This could through associations with arm swing amplitude result in an unexpected effect of stride frequency, and also introduce new thorax-pelvis control parameters, such as pelvis amplitude, that would have no effect on thorax-pelvis relative phase in a second order linear system. Second, the experimental manipulation of step length by Huang et al. (Huang et al., 2011; Huang et al., 2010) affected the frequencies, amplitudes and timing of both axial pelvis rotations and arm swing. If these parameters affect thorax rotations, the effect of the frequency of pelvis rotations might be reduced or even inverted. To provide more insight in the effect of each parameter individually, computer simulation can be used as this enables independent manipulation of each parameter.

In two studies by Prins et al. (2019a; 2019b), a forward dynamic model was introduced to predict the effect of multiple gait parameters on thorax-pelvis relative phase. The model consisted of the passive spring-damper-mass system as described above, with the addition of shoulder reaction forces. Empirically observed pelvis rotations and shoulder reaction forces were imposed on the model, which then predicted time series of thorax rotations. The stiffness and damping of the trunk were estimated using an iterative process (see Prins et al. (2019a)), after which the isolated effect of individual parameters, such as trunk stiffness, could be simulated. The addition of shoulder reaction forces clearly affected the dynamics of the system, compared to a second order system (Figure 4). While the addition of shoulder reaction forces made the model fit the experimental data much better than a second-order linear model without shoulder forces, deviations between modelled and actual behaviour suggest that trunk muscle activity affects thorax rotations in addition to the dynamics modelled.

In the first study (Prins et al., 2019a), it was reported that increased axial trunk stiffness results in more in-phase thorax-pelvis coordination, as expected. However, observed thorax-pelvis relative phase was not predictive of model-based estimates of axial trunk stiffness in a cohort of low back pain patients and healthy 
controls obtained from an earlier study (Prins et al., 2016). Thus, although trunk stiffness affects thorax-pelvis relative phase, it does not appear to be the cause of altered thorax-pelvis relative phase in patients with low back pain. This could partially be explained by arm swing amplitude: the model showed that axial trunk stiffness and arm swing amplitude have opposite effects on thorax-pelvis relative phase (larger arm swing amplitude, more out-of-phase coordination). Model-based estimates of trunk stiffness and experimentally obtained shoulder reaction forces were found to be positively correlated thus counteracting each other's effect on thorax-pelvis relative phase.

Surprisingly, in the study in which pelvis amplitude was manipulated during gait (Prins et al., 2019b), axial trunk stiffness was lower in the condition in which subjects walked with large pelvis amplitudes. In that condition, more inphase thorax-pelvis coordination was observed compared to normal walking, although simulations predicted that a reduction in stiffness would result in more out-of-phase thorax-pelvis coordination (Prins et al., 2019a). It appears that again two opposing mechanisms were at work simultaneously: (1) increased pelvis ROM results in more in-phase thorax-pelvis coordination and (2) lower apparent trunk stiffness results in more out-of-phase thorax-pelvis coordination. Apparently, the effect of increased pelvis ROM was larger than that of reduced stiffness, resulting in a net shift towards more in-phase thorax-pelvis coordination. In line with this interpretation, a forward dynamic simulation of walking with a larger pelvis amplitude without altering axial trunk stiffness had an even larger effect on thoraxpelvis relative phase than observed in the experiment (Prins et al., 2019b).

The independent effects of stride frequency and timing of axial pelvis rotations have been simulated for this review using data from Prins et al. (2016), see Appendix. In contrast to the findings of Huang et al. (2011; 2010), higher stride frequency was predicted to result in more out-of-phase thorax-pelvis coordination. The modelled effect of stride frequency on thoraxpelvis coordination was partially caused by the arms. If arm swing amplitude is kept constant with increasing stride frequency, the accelerations of the arms increase, resulting in larger reaction forces in the shoulders. If the reaction forces in the shoulders are kept constant, higher stride frequency still results in more out-of-phase thorax-pelvis relative phase, although this effect is smaller than in a situation with the more realistic effect of arm swing (Figure 5).

\section{Conclusions}

Thorax-pelvis relative phase at high gait speeds has been found to be more in-phase in some low back pain patients compared to healthy controls but not in all. This finding was initially made in studies motivated by the dynamical systems theory that considered this relative phase as a potential collective variable representing the orderly behavior of the complex motor control system. However, it was found that thorax-pelvis relative phase cannot usefully be considered a collective variable. Nevertheless, the empirical finding of more in-phase coordination was fairly robust, albeit with large between-subject variance, certainly when compared to other aspects of trunk kinematics that are mostly not different between patients and controls (Koch and Hansel, 2018). Subsequent emphasis in the literature therefore shifted from a motivation based on the dynamical systems approach to one based on biomechanics. The change in relative phase was linked to changes specifically in trunk stiffness. We found that thorax-pelvis relative phase is affected by several interacting factors, making it a poor proxy of all of these factors including trunk stiffness. More specifically, the findings presented in this review suggest that pelvis rotations and arm swing are significant drivers of thorax rotations. At high gait speed, the arms and pelvis move outof-phase, causing these drivers to pull the timing of thorax rotations in opposite directions. The timing of thorax rotations shifts towards the timing of a given driver if:

- The amount of force that driver exerts on the thorax increases. This can be caused by increased movement amplitudes of the pelvis or arms, or, for the pelvis, by an increased axial trunk stiffness.

The relative phase between the two drivers becomes smaller (i.e., arm-pelvis relative phase towards zero): when the arms and pelvis move more or less in-phase, thorax timing will be similar to that of these segments (i.e., in-phase with pelvis and arms), when the arms and pelvis are out-of- 
phase, thorax timing will be between that of the pelvis and that of the arms.

The combined observations of higher stride frequency and more in-phase thorax-pelvis coordination (Huang et al., 2010; Lamoth et al., 2006a) might have indirect causes through these mechanisms.

Although recent studies have provided more insight in the potential causes of more inphase thorax-pelvis coordination in low back pain, research questions have shifted rather than that the problem of altered thorax-pelvis coordination during gait has been solved. It seems unlikely that altered trunk coordination as seen in patients with low back pain is the result of splinting or guarding against lumbar movement. Walking with large pelvis rotations appears to be a more likely cause for more in-phase thorax- pelvis coordination in this group. However, the cause or goal of these large pelvis rotations is unknown. Possibly, altered timing or amplitude of hip muscle activation plays a role (Vogt et al., 2001), or flexibility of passive elastic tissues of the hip joint (Hines et al., 2018). The higher stride frequency observed in patients (Lamoth et al., 2006a; Vogt et al., 2001) may also contribute to the more in-phase coordination. In conclusion, thorax-pelvis relative phase cannot be considered as a collective variable reflecting the orderly behaviour of a complex underlying system, nor is it a marker of specific changes in trunk biomechanics. The fact that it is affected by multiple factors could be the cause of considerable between-subject variance of this measure in low back pain patients and healthy controls alike.

\section{Acknowledgements}

SMB was funded by a VIDI grant (016.Vidi.178.014) from the Dutch Organisation for Scientific Research (NWO). Wu WH was supported by the National Natural Science Foundation of China (\#81272161), Natural Science Foundation of Fujian Province, China (\#2019J01470), Research and Development Fund of Quanzhou City (\#Z[2014]0009,\#2017Z007), Programme for Medical Innovation of Fujian Province(\#2019-CX-30), Programme for the Leading Talent of Quanzhou(\#[2014]61) and, Fund of Professorship for Academic Development of Fujian Medical University (\#JS11001).

\section{References}

Beek, P.J., Peper, C.E., Stegeman, D.F., 1995. Dynamical models of movement coordination. Human Movement Science 14, 573-608.

Bruijn, S.M., Meijer, O.G., van Dieen, J.H., Kingma, I., Lamoth, C.J., 2008. Coordination of leg swing, thorax rotations, and pelvis rotations during gait: the organisation of total body angular momentum. Gait Posture 27, 455-462.

Byblow, W.D., Chua, R., Goodman, D., 1995. Asymmetries in Coupling Dynamics of Perception and Action. J Mot Behav 27, 123-137.

Cholewicki, J., Juluru, K., Radebold, A., Panjabi, M.M., McGill, S.M., 1999. Lumbar spine stability can be augmented with an abdominal belt and/or increased intra-abdominal pressure. Eur Spine J 8, 388-395.

Crosbie, J., Vachalathiti, R., Smith, R., 1997. Patterns of spinal motion during walking. Gait Posture 5, 6-12.

Donker, S.F., Beek, P.J., Wagenaar, R.C., Mulder, T., 2001. Coordination between arm and leg movements during locomotion. J Mot Behav 33, 86-102.

Ford, M.P., Wagenaar, R.C., Newell, K.M., 2007. Arm constraint and walking in healthy adults. Gait Posture 26, 135-141.

Gardner-Morse, M., Stokes, I.A.F., 1998. The effects of abdominal muscle coactivation on lumbar spine stability. Spine 23, 86-92.

Gardner-Morse, M.G., Stokes, I.A.F., 2001. Trunk stiffness increases with steady-state effort. Journal of Biomechanics 34, 457-464.

Ghamkhar, L., Kahlaee, A.H., 2015. Trunk Muscles Activation Pattern During Walking in Subjects With and Without Chronic Low Back Pain: A Systematic Review. PM R 7, 519-526.

Graven-Nielsen, T., Lund, H., Arendt-Nielsen, L., Danneskiold-Samsøe, B., Bliddal, H., 2002. Inhibition of maximal voluntary contraction force by experimental muscle pain: A centrally mediated mechanism. Muscle and Nerve 26, 708-712. 
Haken, H., Kelso, J.A., Bunz, H., 1985. A theoretical model of phase transitions in human hand movements. Biol Cybern 51, 347-356.

Hines, M.G., Tillin, N.A., Luo, J., Lee, R., 2018. Passive elastic contribution of hip extensors to joint moments during walking in people with low back pain. Clin. Biomech. 60, 134-140.

Huang, Y., Bruijn, S.M., Lin, J., Meijer, O.G., Wu, W., Abbasi-Bafghi, H., Lin, X., Dieën, J.H.v., 2011. Gait adaptations in low back pain patients with lumbar disc herniation: Trunk coordination and arm swing. Eur Spine J 20, 491-499.

Huang, Y., Meijer, O.G., Lin, J., Bruijn, S.M., Wu, W., Lin, X., Hu, H., Huang, C., Shi, L., Dieën, J.H.v., 2010. The effects of stride length and stride frequency on trunk coordination in human walking. Gait \& Posture 31, 444-449.

Hulbert, S., Ashburn, A., Robert, L., Verheyden, G., 2015. A narrative review of turning deficits in people with Parkinson's disease. Disabil Rehabil 37, 1382-1389.

Kelso, J.A., 1984. Phase transitions and critical behavior in human bimanual coordination. Am J Physiol 246, R1000-1004.

Kelso, J.A., Jeka, J.J., 1992. Symmetry breaking dynamics of human multilimb coordination. J Exp Psychol Hum Percept Perform 18, 645-668.

Kelso, J.A.S., 1981. On the oscillatory basis of movement. Bull Psychonom Soc 18, 63.

Koch, C., Hansel, F., 2018. Chronic Non-specific Low Back Pain and Motor Control During Gait. Front Psychol 9, 2236.

Kubo, M., Holt, K.G., Saltzman, E., Wagenaar, R.C., 2006. Changes in axial stiffness of the trunk as a function of walking speed. J Biomech 39, 750-757.

Lakke, J.P.W.F., 1985. Axial apraxia in Parkinson's disease. J Neurol Sci 69, 37-46.

Lamb, P.F., Stöckl, M., 2014. On the use of continuous relative phase: Review of current approaches and outline for a new standard. Clin. Biomech. 29, 484-493.

Lamoth, C.J., Beek, P.J., Meijer, O.G., 2002a. Pelvis-thorax coordination in the transverse plane during gait. Gait Posture 16, 101-114.

Lamoth, C.J., Daffertshofer, A., Huys, R., Beek, P.J., 2009. Steady and transient coordination structures of walking and running. Hum Mov Sci 28, 371-386.

Lamoth, C.J., Daffertshofer, A., Meijer, O.G., Beek, P.J., 2006a. How do persons with chronic low back pain speed up and slow down? Trunk-pelvis coordination and lumbar erector spinae activity during gait. Gait Posture 23, 230-239.

Lamoth, C.J., Meijer, O.G., Daffertshofer, A., Wuisman, P.I., Beek, P.J., 2006b. Effects of chronic low back pain on trunk coordination and back muscle activity during walking: changes in motor control. Eur Spine J $15,23-40$.

Lamoth, C.J., Meijer, O.G., Wuisman, P.I., van Dieen, J.H., Levin, M.F., Beek, P.J., 2002b. Pelvis-thorax coordination in the transverse plane during walking in persons with nonspecific low back pain. Spine (Phila Pa 1976) 27, E92-99.

Liang, B.W., Wu, W.H., Meijer, O.G., Lin, J.H., Lv, G.R., Lin, X.C., Prins, M.R., Hu, H., Dieën, J.H.v., Bruijn, S.M., 2014. Pelvic step: The contribution of horizontal pelvis rotation to step length in young healthy adults walking on a treadmill. Gait \& Posture 39, 105-110.

Mangone, M., Scettri, P., Paoloni, M., Procaccianti, R., Spadaro, A., Santilli, V., 2011. Pelvis-shoulder coordination during level walking in patients with ankylosing spondylitis. Gait Posture 34, 1-5.

Ng, J.K., Kippers, V., Parnianpour, M., Richardson, C.A., 2002a. EMG activity normalization for trunk muscles in subjects with and without back pain. Medicine and Science in Sports and Exercise 34, 10821086.

Ng, J.K., Richardson, C.A., Parnianpour, M., Kippers, V., 2002b. EMG activity of trunk muscles and torque output during isometric axial rotation exertion: a comparison between back pain patients and matched controls. Journal of Orthopaedic Research 20, 112-121.

Prins, M.R., Bruijn, S.M., Meijer, O.G., van der Wurff, P., van Dieën, J.H., 2016. Mechanical Perturbations of the Walking Surface Reveal Unaltered Axial Trunk Stiffness in Chronic Low Back Pain Patients. PloS one 11, e0157253. 
Prins, M.R., Bruijn, S.M., Meijer, O.G., van der Wurff, P., van Dieën, J.H., 2019a. Axial Thorax-Pelvis Coordination During Gait is not Predictive of Apparent Trunk Stiffness. Sci Rep 9, 1066.

Prins, M.R., Cornellissen, L.E., Meijer, O., van der Wurff, P., Bruijn, S.M., van Dieën, J.H., 2019b. Axial Pelvis Range of Motion Affects Thorax-Pelvis Timing During Gait. J Biomech 95, 109308.

Russell Esposito, E., Wilken, J.M., 2014. The relationship between pelvis-trunk coordination and low back pain in individuals with transfemoral amputations. Gait Posture 40, 640-646.

Schmidt, R.C., Carello, C., Turvey, M.T., 1990. Phase Transitions and Critical Fluctuations in the Visual Coordination of Rhythmic Movements Between People. J Exp Psychol Hum Percept Perform 16, 227247.

Seay, J.F., van Emmerik, R.E.A., Hamill, J., 2011a. Influence of low back pain status on pelvis-trunk coordination during walking and running. Spine 36, E1070-1079.

Seay, J.F., van Emmerik, R.E.A., Hamill, J., 2011b. Low back pain status affects pelvis-trunk coordination and variability during walking and running. Clin. Biomech. 26, 572-578.

Selles, R.W., Wagenaar, R.C., Smit, T.H., Wuisman, P.I., 2001. Disorders in trunk rotation during walking in patients with low back pain: a dynamical systems approach. Clinical Biomechanics 16, 175-181.

Staudenmann, D., Roeleveld, K., Stegeman, D.F., van Dieen, J.H., 2010. Methodological aspects of SEMG recordings for force estimation--a tutorial and review. J Electromyogr Kinesiol 20, 375-387.

Tavernese, E., Paoloni, M., Mangone, M., Castelli, E., Santilli, V., 2016. Coordination between pelvis and shoulder girdle during walking in bilateral cerebral palsy. Clin. Biomech. 32, 142-149.

Van Criekinge, T., Saeys, W., Hallemans, A., Velghe, S., Viskens, P.J., Vereeck, L., De Hertogh, W., Truijen, S., 2017. Trunk biomechanics during hemiplegic gait after stroke: A systematic review. Gait Posture 54, 133-143.

van den Hoorn, W., Bruijn, S.M., Meijer, O.G., Hodges, P.W., van Dieen, J.H., 2012. Mechanical coupling between transverse plane pelvis and thorax rotations during gait is higher in people with low back pain. J Biomech 45, 342-347.

van Dieën, J.H., Reeves, N.P., Kawchuk, G., van Dillen, L.R., Hodges, P.W., 2019a. Analysis of motor control in low-back pain patients, a key to personalized care? J Orthop Sports Phys Ther 49, 380-388.

van Dieën, J.H., Reeves, N.P., Kawchuk, G., van Dillen, L.R., Hodges, P.W., 2019b. Motor control changes in low-back pain: Divergence in presentations and mechanisms. J Orthop Sports Phys Ther 49, 370-379.

Van Emmerik, R.E., Wagenaar, R.C., Winogrodzka, A., Wolters, E.C., 1999. Identification of axial rigidity during locomotion in Parkinson disease. Archives of Physical Medicine and Rehabilitation 80, 186-191.

Vogt, L., Pfeifer, K., Portscher, M., Banzer, W., 2001. Influences of nonspecific low back pain on threedimensional lumbar spine kinematics in locomotion. Spine 26, 1910-1919.

Wagenaar, R.C., Beek, W.J., 1992. Hemiplegic gait: a kinematic analysis using walking speed as a basis. J Biomech 25, 1007-1015.

Wagenaar, R.C., van Emmerik, R.E.A., 1994. Dynamics of pathological gait. Hum Mov Sci 13, 441-471.

Wagenaar, R.C., van Emmerik, R.E.A., 2000. Resonant frequencies of arms and legs identify different walking patterns. J Biomech 33, 853-861.

Wu, W.H., Lin, X.C., Meijer, O.G., Gao, J.T., Hu, H., Prins, M.R., Liang, B.W., Zhang, L.Q., Van Dieen, J.H., Bruijn, S.M., 2014. Effects of experimentally increased trunk stiffness on thorax and pelvis rotations during walking. Hum Mov Sci 33, 194-202.

Wu, W.H., Meijer, O.G., Bruijn, S.M., Hu, H., Dieën, J.H.v., Lamoth, C.J.C., Royen, B.J.v., Beek, P.J., 2008 a. Gait coordination in pelvic girdle pain: Horizontal pelvic and thoracic rotations and their coordination. Eur Spine J 17, 1160-1169.

Wu, W.H., Meijer, O.G., Bruijn, S.M., Hu, H., van Dieen, J.H., Lamoth, C.J., van Royen, B.J., Beek, P.J., $2008 b$. Gait in Pregnancy-related Pelvic girdle Pain: amplitudes, timing, and coordination of horizontal trunk rotations. Eur Spine J 17, 1160-1169. 


\section{Appendix}

A forward dynamic model was used to evaluate the effects of axial trunk stiffness, arm swing amplitude, pelvis amplitude, stride frequency and timing of pelvis rotations relative to the arms on thoraxpelvis relative phase during gait. The details of the model are described in Prins et al. (2019a). In brief, the model predicts axial (around L5S1) thorax rotations from observed axial pelvis rotations, shoulder reaction forces and thorax inertia. Axial trunk stiffness and damping are estimated using an optimization procedure that minimizes the root mean square error between predicted and observed axial thorax rotations. Experimental data of 15 low back pain patients and 15 healthy controls that walked on a treadmill at 4 $(\mathrm{km} / \mathrm{h})$ were used. Details of the experimental procedure are described in Prins et al. (2016).

After estimating the apparent axial trunk stiffness and damping of each subject with the corresponding modelled time series of thorax rotations, the effects of the aforementioned parameters on thorax-pelvis relative phase was assessed. To this aim, simulations were run after multiplication of these parameters by factors of $0.5,0.75,1.25$ and 1.5., assuming that the differences in obtained time series of axial thorax rotations would reflect the effects of these parameters on thorax-pelvis coordination. Increasing stride frequency while keeping arm swing amplitude constant, would increase shoulder reaction forces in the shoulder. When stride frequency was multiplied by a factor $B$, shoulder reaction forces were multiplied by a factor $B^{2}$ (because if $f(t)=\sin (B t)$, then $f^{\prime \prime}(t)=-B^{2} \cos (B t)$ ). To evaluate the contribution of this effect of arm swing, simulations were run with unaltered shoulder reaction forces as well. The effects of these manipulations on thorax-pelvis coordination were quantified by calculating the phase of the frequency response function from pelvis to thorax rotations at the stride frequency. The stride frequency was determined as the local peak in the power spectrum of the pelvis rotations closest to $1(\mathrm{~Hz})$.

The effects of arm swing, axial trunk stiffness and pelvis amplitude were evaluated in a similar manner in previous research (Prins et al., 2019a; Prins et al., 2019b), while the effect of stride frequency and arm-pelvis relative phase had not been assessed before.

\section{Corresponding author:}

\section{Jaap H. van Dieën,}

Department of Human Movement Sciences, Faculty of Behavioural and Movement Sciences, Vrije Universiteit Amsterdam, and Amsterdam Movement Sciences, Amsterdam, The Netherlands

E-mail: j.van.dieen@vu.nl 